\title{
Illustrating COLIBRI VR, an Open-Source Toolkit to Render Real-World Scenes in Virtual Reality
}

\author{
Grégoire Dupont de Dinechin* $\quad$ Alexis Paljic ${ }^{\dagger}$
}

Centre for Robotics, MINES ParisTech, PSL University - Paris, France
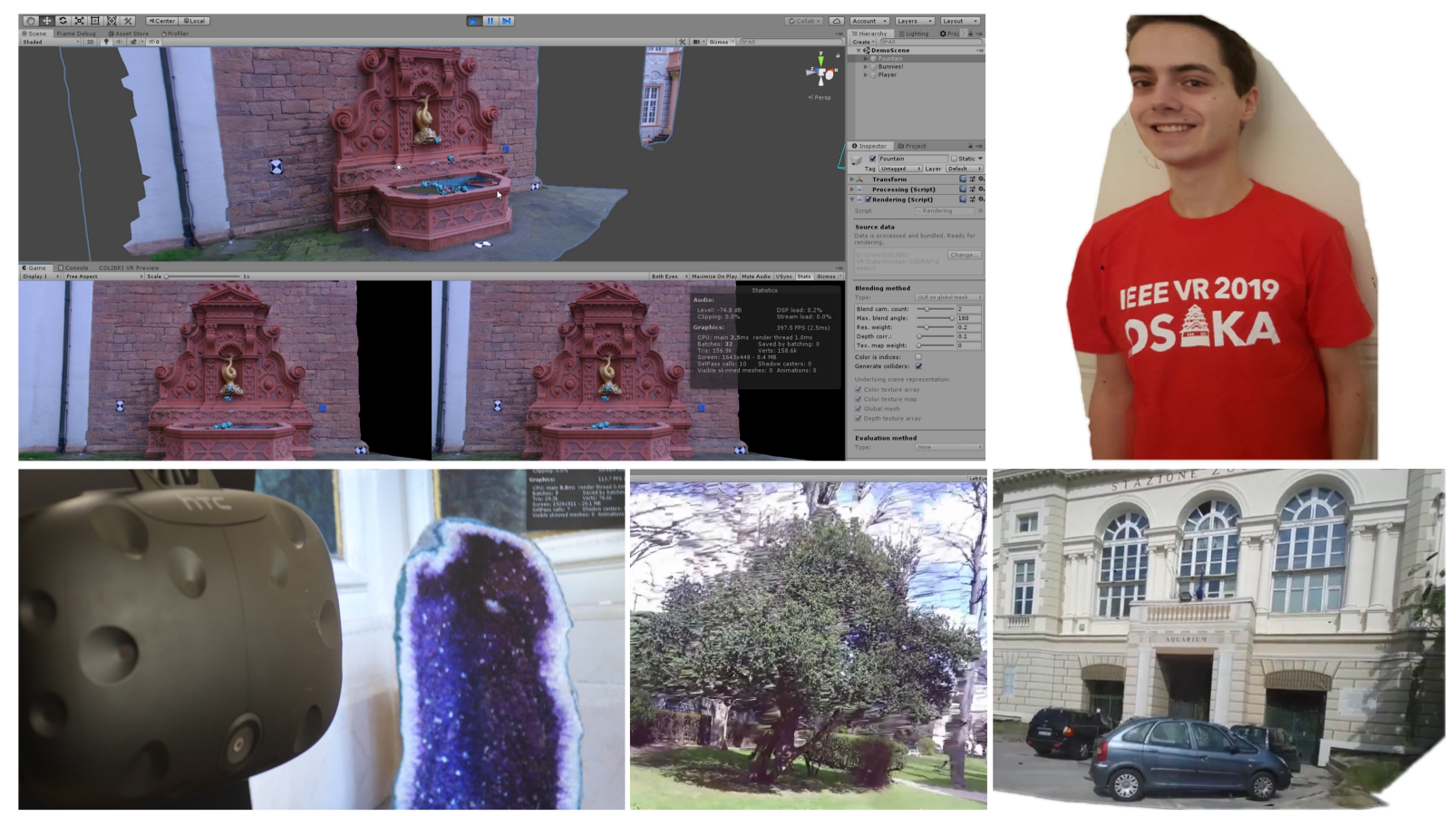

Figure 1: Using COLIBRI VR to render real-world photographs of people, objects, and places in VR, as interactive 3D assets that provide motion parallax and view-dependent rendering effects.

\section{ABstract}

This video submission illustrates the Core Open Lab on Image-Based Rendering Innovation for Virtual Reality (COLIBRI VR), an opensource toolkit we developed to help authors render photographs of real-world people, objects, and places as responsive $3 \mathrm{D}$ assets in VR. We integrated COLIBRI VR as a package for the Unity game engine: in this way, the toolset's methods can easily be accessed from a convenient graphical user interface, and be used in conjunction with the game engine's built-in tools to quickly build interactive virtual reality experiences. Our primary goal is to help users render realworld photographs in VR in a way that provides view-dependent rendering effects and compelling motion parallax. For instance, COLIBRI VR can be used to render captured specular highlights, such as the bright reflections on the facets of a mineral. It also enables providing motion parallax from estimated geometry, e.g. from a depth map associated to a $360^{\circ}$ image. We achieve this by implementing efficient image-based rendering methods, which we optimize to run at high framerates for VR. We make the toolkit openly available online, so that it might be used to more easily learn

*e-mail: gregoire.dupont_de_dinechin@mines-paristech.fr

†e-mail: alexis.paljic@mines-paristech.fr

(C2020 IEEE. Personal use of this material is permitted. Permission from IEEE must be obtained for all other uses, in any current or future media, including reprinting/republishing this material for advertising or promotional purposes, creating new collective works, for resale or redistribution to servers or lists, or reuse of any copyrighted component of this work in other works. about and apply image-based rendering in the context of virtual reality content creation. 